\section{Does workplace counselling work?}

\author{
JOHN McLEOD / MAX HENDERSON
}

Edited and introduced by Mary Cannon, Kwame McKenzie and Andrew Sims.

\section{INTRODUCTION}

There has been a rapid increase in compensation claims for work-related stress in recent years (Dyer, 2002). A Court of Appeal ruling last year (Sutherland $v$. Hatton, 2002) made it clear that employees who feel under stress at work should inform their employers and give them a chance to do something about it. Any employer who offers a confidential counselling service with access to treatment may have some protection from prosecution. In the face of a possible explosion in the provision of such services we need to ask - do they actually work? In 2001 the British Association for Counselling and Psychotherapy commissioned and published a report, Counselling in the Workplace: The Facts (McLeod, 2001), which described itself as 'the most comprehensive possible review of all English language studies of counselling in the workplace'. The results appeared clear and unequivocal. After counselling, workrelated symptoms returned to normal in more than half of all clients and sickness absence was reduced by over $25 \%$. The report has received much publicity in the general medical press (Mayor, 2001). But just how reliable is the evidence? We asked Professor John McLeod, the author of the report, and Dr Max Henderson, Clinical Research Fellow in Occupational Psychiatry, to debate the issue: 'Does workplace counselling work?' The arguments will inform other debates into interventions that seem intrinsically to be a 'good thing' but that have not yet been subjected to rigorous investigation.

\section{FOR}

Workplace counselling may be defined as the provision of brief psychological therapy for employees of an organisation, which is paid for by the employer. An 'external' service, such as an Employee Assistance Programme (EAP), typically comprises face-to-face counselling, a telephone helpline, legal advice and critical-incident debriefing. In an 'in-house' service, counsellors may be directly employed by the organisation. Workplace counselling offers employees a facility that is confidential, easily accessed (initial appointment normally within 2 weeks), provides a properly qualified and supervised practitioner, does not raise the threat of a diagnosis of psychiatric disorder, and promises to alleviate distress within a reasonably short period of time (most services allow only 6-8 sessions in any one year). Workplace counselling offers the employer a service that is valued by employees, has the potential for savings by reducing sickness absence, takes pressure off managers through the availability of a constructive means of dealing with 'difficult' staff or situations, and contributes to its reputation as a caring employer. Workplace counselling is often viewed by employers as an insurance policy against the threat of compensation claims made by employees exposed to work-related stress.

The provision of workplace counselling has steadily expanded over the past 20 years, with more than $75 \%$ of medium and large organisations in Britain and North America making counselling available to their staff (Carroll \& Walton, 1999; Oher, 1999).

A review of research into the outcomes of workplace counselling (McLeod, 2001) identified 34 studies, including controlled studies, naturalistic studies in which reliable pre- and post-counselling data were collected, and case studies. Employees presented to counselling with high levels of psychological symptoms. Those who received counselling were highly satisfied, and believed it had helped them resolve their problem. Clinically significant improvement in levels of anxiety and depression was reported in $60-75 \%$ of clients. Counselling was associated with reduction in sickness absence and improvement in other organisational outcomes such as more positive work attitudes, fewer accidents and enhanced work performance.

It is important to recognise the limitations of the existing research base for workplace counselling (McLeod, 2001). This is a field in which research has been significantly constrained by commercial considerations. There is also a great deal of sensitivity around confidentiality; the fear that 'management' may learn that a person has received counselling has made many clients and counsellors reluctant to complete research questionnaires. High attrition rates are found in such research samples.

However, despite these methodological weaknesses, the general picture that emerges is that workplace counselling is appreciated by its users, and appears to have a positive impact on objective measures of distress (e.g. sickness absence) and on self-reported measures of symptomatology.

Probably, several factors have contributed to the growth and popularity of workplace counselling. At one level, workplace counselling can be viewed merely as an application of methods of brief, relationship-focused psychological intervention that have been shown to be effective in other settings. A distinctive strength of workplace counselling is that the client is seen by a therapist who is sensitised to the combination of personal and work pressures that the person may present. Workplace counselling is a systemic, as well as individual, intervention. The introduction of a counselling service may begin to change the way that managers and other staff think and talk about emotional difficulties and personal problems. The costs to employers of psychological disability are clearly understood (Goldberg \& Steury, 2001). The acceptability of workplace counselling is certainly linked to shifts in the meaning of work, and the movement away from collective to more individual modes of worker resistance (Wainwright \& Calnan, 2002).

In a recent qualitative study, Millar (2002) interviewed police officers and support staff who had received counselling for work-related difficulties. Most of the participants reported that counselling had helped them to overcome the problem that 
had led to them seek help. More striking, though, was the finding that all of these informants described themselves as learning something new and useful about themselves as a result of counselling. For example, an experienced detective stated that 'I am $100 \%$ better at listening now to a person'. Although further controlled studies of the effectiveness of workplace counselling on psychiatric symptoms are undoubtedly necessary, it is also essential to acknowledge the relevance of a wider research agenda (McLeod, 2001), in which the role of workplace counselling can be examined in relation to a range of issues such as health, organisational culture and work effectiveness.

John McLeod Tayside Institute for Health Studies, University of Abertay Dundee, Dudhope Castle, Dundee DD3 6HF, UK. E-mail:

j.mcleod@tay.ac.uk

\section{AGAINST}

Employment practices in the UK are changing, with more people on short-term contracts, working part-time and with less job security. An added burden for employees, employers and the economy as a whole is the large number of employees absent from work, sick or retired because of ill health. Although underrecognised, frank psychiatric conditions - most notably anxiety and depression but also somatoform disorders and substance misuse - account for a growing proportion of these absences.

A new diagnosis has recently emerged which is ubiquitous, not viewed pejoratively, widely used on sickness certificates and has formed the basis of legal claims against employers: workplace stress. It does not seem to have an objective definition and thus any overlap with the psychiatric diagnoses already noted is unclear. Fortunately, workplace stress is apparently treatable; in fact, it is highly responsive to treatment. The name of this well-tolerated and efficacious treatment is workplace counselling.

Work and workplaces are rapidly evolving and 21 st-century occupational medicine will have to manage a different set of problems from its early-20th-century predecessor. However, where previously a link between exposure and illness was clear and such exposure could have occurred only in the workplace, now many complaints have more vague, non-specific symptoms and signs, and multi-factorial aetiology is the norm. The concept of workplace stress implies that work is the causative factor but despite an individual being unhappy at work and using a work-based outlet to complain, symptoms may be explained in several other ways. There may be a perception that appropriate help is difficult to access via more traditional routes. Alternatively, this might represent the well-recognised phenomenon of effort after meaning.

Treatment for such a heterogeneous group of problems with a single therapeutic modality would seem almost impossible, yet this is what workplace counselling claims to do. The quality of the evidence to support this is poor. Professor McLeod, in his recent review (McLeod, 2001), failed to find a single study showing a negative outcome for workplace counselling - a clear case for suspecting publication bias, yet an issue barely touched upon in the review. All treatments that work have the potential for adverse effects and this holds true for psychotherapeutic as well as pharmacological interventions. The available studies are limited by small sample sizes, short follow-up periods and wide variation in the form and content of the therapy given. Even in the 'best evidence' section only 5 out of 19 studies had a form of non-treatment control group. Of these, two used controls who were not seeking any form of help at all and one used those who dropped out at the first session as controls.

The arguments for randomised controlled trials are well-rehearsed and no less applicable to a psychological than to a physical treatment. Non-randomised studies are more likely to produce false positive results (Chalmers et al, 1983). The medical literature contains numerous examples of treatments that were felt to be beneficial but when subjected to randomised controlled trials were shown to be ineffective or, worse still, harmful. Especially in a situation where there is a strong possibility the complaint may get better spontaneously (as so many do) there is an onus on researchers to demonstrate the additional benefits their treatment brings. To date, such evidence just is not available for workplace counselling, certainly removing the argument that randomisation is unethical. The medicalisation or 'psychologisation' of normal human distress is a real and unwelcome possibility. So too is the possibility of failing to manage the other factors involved; work may indeed be a part of the jigsaw but in all probability only a part - most stressors are external to work.
So does workplace counselling work? Well, that depends on what you want it to do. Many studies highlight the significant client satisfaction associated with workplace counselling. If that is its aim, then it succeeds. Studies in closely related fields (e.g. cognitive-behavioural therapy $v$. relaxation for chronic fatigue syndrome) attest to the discordance between simple measures of satisfaction and clinical outcome (Deale et al, 1997). What data there are on workplace counselling do not allow us to conclude that satisfaction is allied with meaningful clinical improvement or even consistent employment objectives such as reduced absenteeism. The cynic might suggest that if employers want a tool that their employees appreciate and that, in view of the recent Court of Appeal ruling (Sutherland v. Hatton, 2002), protects them from litigation, then workplace counselling does indeed work. The objective observer looking at clinical outcomes would undoubtedly conclude that there is no reliable evidence that workplace counselling is of benefit.

Max Henderson Clinical Research Fellow in Occupational Psychiatry, Guys', Kings' and St Thomas' Medical School, Academic Department of Psychological Medicine, 103 Denmark Hill, London SE5 8AF, UK. E-mail: m.henderson@iop.kcl.ac.uk

\section{REFERENCES}

Carroll, M. \& Walton, M. (eds) (1999) Handbook of Counselling in Organisations. London: Sage.

Chalmers, T., Celano, P., Sacks, H., et al (1983) Bias in treatment assignment in controlled clinical trials. New England Journal of Medicine, 309, 1358-1361.

Deale, A., Chalder, T., Marks, l., et al (1997) Cognitive behavior therapy for chronic fatigue syndrome: a randomized controlled trial. American Journal of Psychiatry, 154, 408-4I4.

Dyer, C. (2002) Judges curb stress cases. Appeal court rulings scrap compensation awards and put onus on workers to tackle employers. The Guardian, 6 February. Goldberg, R. \& Steury, S. (200I) Depression in the workplace: costs and barriers to treatment. Psychiatric Services, 52, 1639-1643.

McLeod, J. (200I) Counselling in the Workplace: The Facts. A Systematic Study of the Research Evidence. Rugby: British Association for Counselling and Psychotherapy.

Mayor, J. (200I) Review confirms workplace counselling reduces stress. BMJ, 322, 637 .

Millar, A. (2002) Beyond resolution of presenting issues: clients' experiences of an in-house police counselling service. Counselling and Psychotherapy Research, 2, 159-166.

Oher, J.M. (ed.) (1999) The Employee Assistance Handbook. New York: Wiley.

Wainwright, D. \& Calnan, M. (2002) Work Stress: The Making of a Modern Epidemic. Buckingham: Open University Press.

Sutherland v. Hatton (2002) EWCA Civ 76. 